\title{
The development of tools to measure the food environment of adolescents
}

\author{
A. A. Lake ${ }^{1,2}$, R. L. Tyrrell ${ }^{2}$, F. Greenhalgh ${ }^{2}$, E. Stamp ${ }^{2}$, M. White ${ }^{2}$, J. C. Mathers ${ }^{2}$ and A. J. Adamson ${ }^{2}$ \\ ${ }^{1}$ Applied Biosciences, School of Applied Sciences, Northumbria University, NE1 8ST, UK and ${ }^{2}$ Human Nutrition Research \\ Centre, Newcastle University NE2 4HH, UK
}

It is hypothesised that the food environment influences eating behaviour and contributes to excessive intakes, which contribute to the aetiology of obesity. However, there has been little research on the characteristics of individual food environments and the research field is constrained by the lack of reliable tools for measuring individual food environments. This research aimed to develop methods and tools to characterise and quantify individual food environments and to measure the impact of the food environment on dietary intakes of adolescents.

Three methodological studies were conducted and four tools - a home food environment questionnaire and three measuring food environment (MFE) tools (for shops, cafés/restaurants and vending machines) - were developed. These tools aimed to measure the availability and variety of food, levels of nutritional information provided and barriers and facilitators to healthful choices. Four-day food diaries were used to record food intake, timing and location of food consumption and the type and number of environments from which each adolescent obtained food. Each environment was visited and assessed by two independent researchers, classified ${ }^{(1)}$, and scored using the tools developed in this project. Inter-rater reliability when using the tools was also assessed. All foods recorded in respondent's 4-d diaries were assigned to the environment from where food was obtained.

The first study developed the tools and methods, the results presented focus on studies 2 and 3 only. Respondents ( $n$ 42, mean age 17.7 years) obtained food from 'home' and from 136 other food environments; 81 outlets were visited and scored by the researchers. Outlet scores were divided into three groups; 20-39.9 ( $n$ 29), 40-49.9 ( $n$ 26) and 50-59.9 ( $n$ 26). The maximum possible score was 100 and a higher score was indicative of a healthier environment. Outlet score groups were compared with the nutrient data from food obtained from that outlet, using a Kruskal-Wallis test. Trends were observed in that foods obtained and consumed by respondents from environments with higher scores were higher in fruits and vegetables, \% energy from protein, protein ( $\mathrm{g}$ ), NSP, Fe, vitamin C and folate and lower in \% energy saturated fat, carbohydrate and NME sugars. However, only for vegetables did this difference between scored environments reach significance $(P=0.012)$, with more vegetables being obtained and consumed from higher scoring food environments. Inter-rater reliability for the tools was high and improved between studies.

These studies have begun to explore nutrient intake within an environmental context. While tools show good inter-rater reliability, further development of the tools and larger sample sizes are required to fully explore this relationship. The identification and characterisation of the food environments to which individual adolescents were exposed begins to provide insight into the environmental context of food choices made at this important life-stage. Work is underway to further develop these tools with the aim of providing a definitive characterisation of the relationship between food environment and body weight in adolescents.

1. Lake AA, Burgoine T, Greenhalgh F et al. (2010) The foodscape: classification and field validation of secondary data sources. Health Place 16, $666-673$. 